Physics Vol. 2, No. 4, pp. 165-184, 1966. Physics Publishing Co. Printed in Great Britain.

\title{
ON THE THEORY OF ELECTROMAGNETIC WAVES IN METALS IN A MAGNETIC FIELd
}

\author{
E. A. KANER and V.G. SKOBOV \\ (Received 1 October 1965 )
}

\begin{abstract}
Summary
Theoretically investigated in the paper is the possibility of the propagation in metals, of electromagnetic waves whose wavelength is small compared to the diameter of the electron orbits in a magnetic field. The dissipative conductivity due to the Cerenkov absorption of the wave by electrons fluctuates with the change in the relationship between the wavelength and the diameter of the electron orbits ("geometric resonance"). The Cerenkov absorption may vanish at the minimums of these oscillations in metals with a simply-connected Fermi surface. The non-dissipative conductivity hence turns out to be greater than the dissipative conductivity due to electron scattering. Consequently, electromagnetic waves with a discrete wave vector and frequency spectrum may be propagated in a metal. The spectrum, damping and polarization of these waves are investigated. It is shown that their frequencies may be both greater and less than the reciprocal of the electron relaxation time. As the magnetic field changes, the surface impedance of the metal experiences resonant oscillations due to the coincidence of the proper frequencies $\omega_{n}$ and the external wave frequency $\omega$.
\end{abstract}

\section{Introduction}

Konstantinov and Perel' [1] first turned attention to the fact that a radio wave penetrates deeply into a metal in the presence of a strong magnetic field $\mathbf{H}$ perpendicular to the surface. In this case the wave vector $\mathbf{k}$ is directed along $\mathbf{H}$ and the Cerenkov absorption associated with the spatial dispersion is lacking because of symmetry (the wave is transverse, and the transverse conductivity is due to the Hall drift and is not associated with energy dissipation). Hence, helicon electromagnetic waves with a quadratic spectrum may be propagated in metals with unequal electron and "hole" concentrations. Later, Buchsbaum and Galt [2] showed that Alfven waves, analogous to those existing in a rarefied plasma, may also be propagated along $\mathbf{H}$ in metals with equal concentrations of carriers of different sign.

The authors [3] have examined the case of arbitrary orientation of the vectors $\mathbf{k}$ and $\mathbf{H}$, when there is, generally, strong absorption of the waves by electrons moving cophasally (Landau damping). It has been shown that rotation of the electrons does not permit them to move strictly cophasally with the wave, and radically diminishes the Landau damping if the sizes of the electron orbits are small compared to the wavelength. Consequently, the damping of the helicon wave, due to Cerenkov electrons, turns out to be on the order of the ratio of the Larmor radius of the electron $R$ to the wavelength, and is small in the case of a sufficiently strong magnetic 
field $(k R \ll 1)$.

The question of the possibility of the propagation of electromagnetic excitations with $a^{\prime}$ wavelength considerably less than the Larmor radius ( $k R \gg 1$ ) in metals, is quite a good deal more complex. The reason is that spatial dispersion and its associated Landau damping play a very essential part in this wavelength range. Hence, shortwave excitations may not generally be propagated in metals. However, under certain conditions, in $k$-space small domains appear in which the wave damping is slight. This is connected with the fact that the Landau damping may experience sharp oscillations depending on the relationship between $R$ and $1 / k$. The physical nature of these oscillations is substantially the same as in the case of the well-known "geometric resonance" in the absorption of ultrasound [4].

Landau damping is determined by electrons for which the phase relations

$$
k_{z} v_{z}-N \Omega=\omega \quad(N=0, \pm 1, \pm 2, \ldots)
$$

are satisfied. Here $\omega$ is the frequency of the wave, $\Omega=e H / m c$ the cyclotron frequency, $e$ the magnitude of the electron charge, $m$ the effective mass, $c$ the velocity of light, $k_{z}$ and $v_{z}$ the projections of the wave vector and electron velocity in the direction of the magnetic field $\mathbf{H}$, the $z$ axis.

The contribution of the electrons with different $N$ to absorption depends essentially on the relationship between their orbit diameters and the wavelength. This is explained as follows. An electron interacts most effectively with an electromagnetic field on those portions of its orbit in which it moves along an equal-phase plane of the wave. In the case of a closed convex electron orbit, there are two such sections (in the neighborhood of the points $A$ and $B$, see. Fig. 1). As the electron moves between these sections, a rapidly oscillating field of the wave

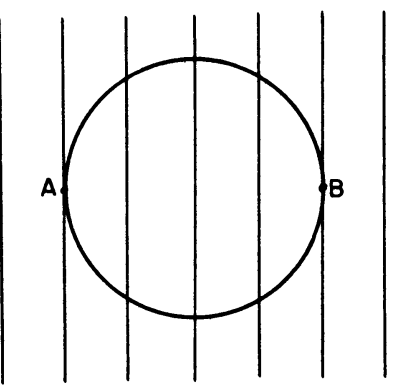

FIGURE 1

acts on it, not changing its energy on the average. Hence, the energy absorbed by the electron depends on the difference in the phases of the wave field at the points $A$ and $B$ at which the electron moves oppositely. If there is a small number of odd half-waves in the orbit diameter, the wave field acts identically on the electron on both sections of the orbit, and the absorption is great. If there is an even number of half-waves in the diameter, then the effect of the wave field on these sections turns out to be opposite, and the absorption is very small.

Condition (1.1) is satisfied for many $N\left(N \sim k_{z} R \gg 1\right)$ when $k_{z} \gg 1 / R$. Hence, among the different groups of electrons there are always such whose contribution to the absorption will be great. Consequently, the oscillation amplitude is relatively small (on the order of the reciprocal of the square root of the number of such groups). If $k_{z} R$ is small, condition (1.1) is satisfied only for $N=0$. In this case the "geometric resonance" oscillations are large [5], 
where the minimum value of the damping is inversely proportional to the mean free path of the electron. It has been shown in [3] that under definite conditions the minimum value of the dissipative current becomes so small that the non-dissipative "Hall" current starts to play a fundamental part. Hence, propagation of electromagnetic waves with a discrete frequency and wave-vector spectrum turns out to be possible.

The low frequency case $\omega \ll v$ ( $v$ the collision frequency of the electron and the scatterers) was examined in [3]. The high frequency domain $\omega \gg v$ was not investigated. Meanwhile, new electromagnetic waves, possessing slight damping, arise in a metal for $\omega \gg v$. The present paper is devoted to an exposition of the results of these investigations.

\section{Formulation of the Problem}

The propagation of a plane monochromatic wave in an unlimited metal is described by the Maxwell equations

$$
k^{2} E_{i}-k_{i} k_{j} E_{j}=\frac{4 \pi i \omega}{c^{2}} \sigma_{i j}(\mathbf{k}, \omega, \mathbf{H}) E_{j}
$$

where $\sigma_{i j}(k, \omega, H)$ are the Fourier components of the conductivity tensor taking account of spatial and time dispersions, as well as the dependence on the permanent magnetic field $\mathbf{H}$; summation from 1 to 3 is understood by the double vector subscripts. We have neglected bias currents, consequently, the condition of electrical quasineutrality of the metal follows from (2. 1):

$$
k_{i} \sigma_{i j} E_{j}=0
$$

Let us select an $x y z$ coordinate system such that the $z$ axis is parallel to the permanent magnetic field $\mathbf{H}$, and the $x$ axis is orthogonal to the vectors $\mathbf{k}$ and $\mathbf{H}$. Later we shall also need a $x \eta \zeta$ coordinate system in which the $\zeta$ axis is parallel to the wave vector k. Moreover, we shall consider that the angle between the $y$ and $\zeta$ axes equals $\pi-\phi$, where

$$
\varphi<1
$$

and therefore,

$$
k_{x}=0, \quad k_{y}=-k, \quad k_{z}=k \Phi
$$

The general expression for the elements of the conductivity tensor, obtained in [3] by using the kinetic equation, will be written in the case of an isotropic electron dispersion law $\varepsilon(\mathbf{p})=\mathbf{p}^{2} / 2 m$ :

$$
\begin{aligned}
& \sigma_{i j}(\mathbf{k}, \omega, \mathbf{H})=\frac{3}{4 \pi} \frac{n_{0} e^{2}}{m \Omega v^{2}} \int_{0}^{\pi} d \theta \sin \theta \int_{0}^{2 \pi} d \tau v_{i}(\theta, \tau) . \\
& \cdot \int_{-\infty}^{T} d \tau^{\prime} v_{j}\left(\theta, \tau^{\prime}\right) \exp \left[\frac{v-i \omega}{\Omega}\left(\tau^{\prime}-\tau\right)+\frac{i \mathbf{k}}{\Omega} \int_{\tau}^{\tau^{\prime}} \mathbf{T}\left(\theta,{T^{\prime \prime}}^{\prime \prime} d \tau^{\prime \prime}\right] .\right.
\end{aligned}
$$

Here $n_{0}$ is the electron concentration; $\quad \mathbf{v}$ the velocity on the Fermi surface $p^{2} / 2 m=\mu ; T$ and $\theta$ 
the azimuthal and polar angles in velocity space. The projections of the electron velocity $\mathbf{v}$ equal

$$
\begin{gathered}
v_{x}(\theta, \tau)=v \sin \theta \cos T_{,} \quad v_{y}(\theta, \tau)=v \sin \theta \sin \tau_{,} \\
v_{z}(\theta)=v \cos \theta .
\end{gathered}
$$

The wave spectrum, damping and polarization are determined from the homogeneous system (2.1). It is convenient to write the condition that its determinant be zero by using (2.2). Let us express the longitudinal field component $E_{\zeta}$ from (2.2) in terms of $E_{x}$ and $E_{\zeta}$ :

$$
E_{\zeta}=-\frac{1}{\sigma_{\zeta \zeta}}\left(\sigma_{\zeta x} E_{x}+\sigma_{\zeta \eta} E_{\eta}\right)
$$

Substituting (2.6) into (2.1), let us represent the equation for the transverse components as

$$
E_{\alpha}=\frac{4 \pi i \omega}{k^{2} c^{2}}\left(\sigma_{\alpha \beta}-\frac{\sigma_{\alpha \zeta \sigma} \zeta \beta}{\sigma_{\zeta \zeta}}\right) E_{\beta}, \quad(\alpha, \beta=x, \eta)
$$

where the index of summation $\beta$ runs through the values $x$ and $\eta$. Therefore, the transverse field components are determined by the "renormalized" tensor

$$
\tilde{\sigma}_{\alpha \beta}=\sigma_{\alpha \beta}-\frac{\sigma_{\alpha \zeta} \sigma \beta}{\sigma_{\zeta \zeta}}
$$

Henceforth, the Latin subscripts $i, j$ will take on values 1 to 3 throughout, and the Greek subscripts $\alpha, \beta$ the values 1 and 2 .

The condition that the determinant of the system (2.7) be zero is:

$$
\left(\frac{k^{2} c^{2}}{4 \pi \omega}\right)^{2}-\left(\tilde{\sigma}_{x x} \tilde{\sigma}_{\eta \eta}-\tilde{\sigma}_{x \eta} \tilde{\sigma}_{\eta x}\right)-i \frac{k^{2} c^{2}}{4 \pi \omega}\left(\tilde{\sigma}_{x x}+\tilde{\sigma}_{\eta \eta}\right)=0 .
$$

\section{Asymptotic Behavior of the Conductivity Tensor}

In order to determine the properties of the electromagnetic waves in the case of large $k R$, it is necessary to find an asymptotic expression for the conductivity tensor $\sigma_{i j}(\mathbf{k}, \omega, \mathbf{H})$. As has been remarked above, we consider the angle between the vectors $H$ and $\mathbf{k}$ to be almost $\pi / 2$ so that $k_{z} R \ll 1$. Nevertheless, we shall assume that the spatial inhomogeneity of the highfrequency field is strong along $\mathbf{H}$. In other words, we are interested in the asymptotic behavior of the tensor $\sigma_{i j}$ under compliance with the following conditions

$$
|v-i \omega| \ll k_{z} v \ll \Omega<k v
$$

It is convenient to carry out the calculations in the $x \eta \zeta$ coordinate system. Let us integrate by parts with respect to $T$ or $\tau^{\prime}$ in the elements of the tensor $\sigma_{i j}$ in (2.4), in which there is a subscript $\zeta$. For example, in the element 


$$
\sigma_{x} \zeta=\frac{3 n_{0} e^{2}}{4 \pi m \Omega v^{2}} \int_{0}^{\pi} \sin \theta d \theta \int_{0}^{2 \pi} v_{x}(\theta, \tau) u_{\zeta}(\theta, \tau) d \tau
$$

where

$$
u_{\zeta}=\frac{Q}{i k} \int_{-\infty}^{T} d T^{\prime} \exp \left[\frac{\nu-i \omega}{\Omega}\left(\tau^{\prime}-T\right)\right] \frac{d}{d \tau^{\prime}} \exp \left[\frac{i k}{\Omega} \int_{T}^{T^{\prime}} v \zeta\left(\theta, T^{\prime \prime}\right) d T^{\prime \prime}\right]
$$

Integrating by parts in (3.3), we obtain

$$
u_{\zeta}=\frac{\Omega}{i k}+\frac{\omega+i v}{k} \int_{-\infty}^{T} d \tau^{\prime} \exp \left[\frac{v-i \omega}{\Omega}\left(\tau^{\prime}-\tau\right)+\frac{i k}{\Omega} \int_{T}^{\tau^{\prime}} v\left(\theta, T^{\prime \prime}\right) d \tau^{\prime \prime}\right]
$$

Upon substituting (3.4) into (3.2), the member with $\Omega / i k$ vanishes identically. The second member in (3.4) may be converted to the form:

$\frac{(\omega+i v) / k}{1-\exp \left[-\frac{2 \pi}{\Omega}\left(\nu-i \omega+i k_{z} v_{z}\right)\right]} \int_{\tau-2 \pi}^{T} d \tau^{\prime} \exp \left[\int_{\tau}^{\tau} \frac{\nu-i \omega+i k \cdot v\left(\theta, \tau^{\prime \prime}\right)}{\Omega} d \tau^{\prime \prime}\right]$.

Now using the inequality (3.1) and neglecting terms on the order of $\left(v-i \omega+i k_{z} v_{z}\right) / \Omega$ and $\phi^{2}$. let us write (3.5) as

$$
\frac{\omega+i v}{k} J_{0}(k R \sin \theta) \frac{\Omega \exp (-i k R \sin \theta \cos \tau)}{v-i \omega+i k_{z} v \cos \theta} .
$$

where $J_{0}$ is the zero-order Bessel function. Substituting (3.6) into (3.2) and integrating with respect to $T$, we find

$$
\sigma_{x} \zeta=\frac{3}{2} \frac{n_{0} e^{2}}{m} \frac{v-i \omega}{k v} \int_{0}^{\pi} \frac{J_{0}(k R \sin \theta) J_{1}(k R \sin \theta) \sin ^{2} \theta d \theta}{v-i \omega+i k_{z} v \cos \theta}
$$

The remaining elements of the tensor $\sigma_{i j}$ may be transformed analogously. As a result, the expression for the conductivity tensor becomes (in the $x \eta \zeta$ coordinate system):

$$
\sigma_{i j}(\mathbf{k}, \omega, \mathbf{H})=\frac{3}{2} \frac{n_{0} e^{2}}{m} \int_{0}^{\pi} \frac{\sin \theta d \theta}{v-i \omega+i k_{z} v \cos \theta}
$$

$$
\times\left[\begin{array}{c}
J_{1}^{2}(x) \sin ^{2} \theta ; \quad-i J_{0}(x) J_{1}(x) \sin \theta \cos \theta ; \quad J_{0}(x) J_{1}(x) \frac{v-i \omega}{k v} \sin \theta \\
i J_{0}(x) J_{1}(x) \sin \theta \cos \theta ; \quad J_{0}^{2}(x) \cos ^{2} \theta ; \quad i J_{0}^{2}(x) \frac{v-i \omega}{k v} \cos \theta \\
-J_{0}(x) J_{1}(x) \frac{v-\imath \omega}{k v} \sin \theta ; \quad i J_{0}^{2}(x) \frac{v-i \omega}{k v} \cos \theta ; \\
\left(\frac{v-i \omega+i k z \cos \theta}{k v}\right)^{2}-J_{0}^{2}(x)\left(\frac{v-i \omega}{k v}\right)^{2}
\end{array}\right]
$$


where $x=k R \sin \theta$. Let us note that because of the smallness of the angle $\varphi$, the $\eta$ and $z$ and $\zeta$ and $-y$ axes may not be differentiated in (3.8).

To simplify (3.8) further, let us use the asymptotic form of the Bessel functions for large values of the argument

$$
\begin{aligned}
& J_{n}(x) \approx\left(\frac{2}{\pi x}\right)^{1 / 2} \cos \left(x-\frac{\pi}{4}-\frac{\pi n}{2}\right) \\
& x>1
\end{aligned}
$$

Let us substitute (3.9) into (3.8), and let us integrate with respect to $\theta$. After sufficiently prolonged calculations (see the Appendix), the asymptotic expression for $\sigma_{i j}$ may be represented as

$$
\begin{aligned}
& \sigma_{i j}(\mathbf{k}, \omega, \mathbf{H})=\frac{3}{2} \frac{n_{0} e^{2}}{m k_{z} v k R} \\
& \times\left[\begin{array}{ccc}
1-F ; & \frac{1}{\sqrt{\pi k R}} \cos \left(2 k R-\frac{\pi}{4}\right)-\frac{v-i \omega}{k_{z} v} G ; & -\frac{v-i \omega}{k v} G \\
-\frac{1}{\sqrt{\pi k R}} \cos \left(2 k R-\frac{\pi}{4}\right)+\frac{\nu-i \omega}{k_{z} v} G ; \quad \frac{v-i \omega}{k_{z} v} ; & \frac{v-i \omega}{k v} \\
\frac{v-i \omega}{k v} G ; \quad \frac{v-i \omega}{k v} ; & 2 k_{z} R \frac{v-i \omega}{k v}
\end{array}\right]
\end{aligned}
$$

where

$$
\begin{aligned}
& F=\frac{2}{\sqrt{\pi}} \int_{0}^{\infty} d x \sin \left(2 k R-\frac{\pi}{4}+x^{2}\right) \exp \left(-2 x \sqrt{k R} \frac{v-i \omega}{k_{z} v}\right), \\
& G=\frac{2}{\sqrt{\pi}} \int_{0}^{\infty} d x \cos \left(2 k R-\frac{\pi}{4}+x^{2}\right) \exp \left(-2 x \sqrt{k R} \frac{v-i \omega}{k_{z} v}\right) .
\end{aligned}
$$

We neglected small terms on the order of $(k R)^{-\frac{1}{2}}$ and $(v-i \omega) / k_{z} v$ in $(3.10)$.

It follows from (3.10) that the magnitude of the longitudinal electric field component $E_{\zeta}$ is considerably greater than the transverse components $E_{x}$ and $E_{\eta}$ :

$$
E_{\zeta} \approx-E_{y}=-\frac{1}{2 k_{z} R}\left(G E_{x}+E_{z}\right) \text {. }
$$


However, despite the relatively large value of $E_{\zeta}$, the "renormalization" (2.8) of the twodimensional tensor $\sigma_{\alpha \beta}$ plays no essential part. The reason is that the elements of $\sigma_{\alpha \zeta}(\alpha=x, \eta)$ are very small and

$$
\tilde{\sigma}_{\alpha \beta} \simeq \sigma_{\alpha \beta} \quad(\alpha, \beta=\dot{x}, \eta) .
$$

It follows from (3.11), (3.12) that the asymptotic behavior of the elements $\sigma_{\alpha \beta}$ depend on the magnitude of the parameter

$$
w=\sqrt{k R} \frac{v-i \omega}{k_{z} v} .
$$

When

$$
|w| \ll 1
$$

we have

$$
\begin{gathered}
\sigma_{x x}=\frac{3 n_{0} e^{2}}{2 m k_{z} v k R}\left[1-\sin 2 k R+\frac{2}{\sqrt{\pi}} w \cos \left(2 k R-\frac{\pi}{4}\right)\right], \\
\sigma_{x z}=-\sigma_{z x}=\frac{3 n_{0} e^{2}}{2 m k_{z} v} \frac{\pi}{(\pi k R)^{3 / 2}}\left[\cos \left(2 k R-\frac{\pi}{4}\right)-\sqrt{\pi} w \cos 2 k R\right] .
\end{gathered}
$$

The real part of the diagonal element $\sigma_{x x}$ is responsible for dissipation of the wave energy. It is a sharply oscillating function of $k R$, taking on its minimum value for

$$
k R=\alpha_{n} \equiv(n+1 / 4) \pi, \quad n=0,1,2, \ldots
$$

These oscillations of Re $\sigma_{x x}$ are due to the fact that electrons moving cophasally with the wave play a fundamental part in the absorption. The longitudinal velocity of these electrons is

$$
v_{z} \simeq|\omega+i v| / k_{z},
$$

and the spread in their orbit diameters is

$$
\delta D \simeq 2 R\left(1-\sqrt{1-\frac{\delta v_{z}^{2}}{v^{2}}}\right) \simeq R \frac{\delta v_{z}^{2}}{v^{2}} .
$$

Condition (3.16) is sufficient for the spread in the diameters of these effective electrons to be small compared with the electromagnetic wavelength since

$$
k \delta D \simeq|w|^{2}
$$

Hence, in the case (3.16) all the effective electrons are under identical conditions relative to the wave. The most essential interaction between the electrons and the wave occurs on those potions of the trajectories where they move along equal-phase planes of the wave, $A$ and $B$ (see Fig. 1). The difference in the wave phase at the points $A$ and $B$ is determined by the quantity $2 k R$. Hence, the quantity $\sigma_{x x}$ oscillates sharply with changes in $k R$. 
In the reverse limiting case

$$
|w| \gg 1
$$

there is a large spread in the orbit diameters of the effective electrons per wavelength, and because of the averaging of their contributions, the amplitude of the oscillations of $\sigma_{x x}$ and $\sigma_{x z}$ is radically decreased

$$
\begin{aligned}
& \sigma_{x x}=\frac{3 n_{0} e^{2}}{2 m k_{z} v k R}\left[1-\frac{1}{\sqrt{\pi} w} \sin \left(2 k R-\frac{\pi}{4}\right)\right] . \\
& \sigma_{x z}=\frac{3 n_{0} e^{2}}{2 m k_{z} v} \frac{\pi}{(\pi k R)^{3 / 2}} \frac{\sin (2 k R-\pi / 4)}{2 w^{2}} .
\end{aligned}
$$

The magnitude of the non-diagonal element $\sigma_{x z}$ hence turns out to be much less than $\sigma_{x x}$ and $\sigma_{z z}$.

\section{Low-frequency Waves with Discrete Spectrum}

Let us first consider electromagnetic wave propagation in the limiting low-frequency case

$$
\omega<v \text {. }
$$

The asymptotic expressions for the elements of the conductivity tensor, obtained in the previous section, are valid for an arbitrary relationship between $\omega$ and $v$. Of greatest interest is analysis of the case (3.16). Let us compare the minimum value of $\sigma_{x x}$ with the magnitude of $\sigma_{x z}$. For $k R=\alpha_{n}$

$$
\left|\frac{\sigma_{x}^{\min }}{\sigma_{x z}}\right|=\frac{2 v}{\phi \Omega}
$$

If this ratio is small, i.e. if the angle $\phi$ satisfies the inequalities

$$
\frac{v}{\Omega} \ll \varphi<\frac{1}{k R}
$$

then the non-dissipative "Hall" conductivity $\sigma_{x z}$ turns out to be considerably greater than the dissipative terms $\sigma_{x x}^{m i n}$ and $\sigma_{z z}$. In other words, the effective dielectric constant of the electron gas becomes greater than the dissipative conductivity for values of the wave vector

$$
k_{n}=\frac{\alpha_{n}}{R}
$$

Hence, propagation of weakly damped electromagnetic waves with a discrete spectrum turns out to be possible in a metal [3]. Admissible values of the wave number $k_{n}$ are determined by the relationship (4.4). Such "quantization" under the particularly classical conditions $\hbar \Omega \ll T$ is due to the fact that the large Landau damping vanishes for $k=k_{n}$. The spectrum of the 
corresponding frequencies $\omega_{n}$ should be found from the general dispersion equation (2.9), in which the wave number $k$ should be replaced by $k_{n}$. Neglecting small terms with $\sigma_{x x}^{\text {min }}$ and $\sigma_{z z}$ in (2.9), we obtain

$$
\omega_{n}=\frac{k_{n}^{2} c^{2}}{4 \pi\left|\sigma_{x z}\left(k_{n}\right)\right|} \equiv \frac{2}{3} \sqrt{2 \pi} \frac{v_{A}{ }^{2}}{v^{2}} \alpha_{n}{ }^{9 / 2} \phi \Omega
$$

where

$$
v_{A}=\frac{c \Omega}{\omega_{0}}=\frac{H}{\sqrt{4 \pi n_{0} m}} .
$$

The wave damping is due to the dissipative conductivity $\sigma_{x x}$. We find the relative damping decrement from (2.9):*

$$
\Gamma_{n}(k)=-\frac{\operatorname{Im} \omega}{\omega_{n}}=\frac{\sigma_{x x}(k)}{2\left|\sigma_{x z}\left(k_{n}\right)\right|}=\frac{\nu}{\phi \Omega}+\sqrt{2 \pi \alpha_{n}} \sin ^{2}\left(k R-\alpha_{n}\right) .
$$

The damping is a minimum for $k R=\alpha_{n}$ and increases sharply as $\left|k R-\alpha_{n}\right|$ increases. The width of the domain in $k$-space in which the weakly-damped wave (4.5) exists is determined from the condition

$$
\Gamma_{n}(k) \ll 1
$$

or

$$
\left(k R-\alpha_{n}\right)^{2} \ll\left(2 \pi \alpha_{n}\right)^{-1 / 2} .
$$

Let us now clarify the nature of the electric field polarization in the wave. Substituting the frequency and wave-vector spectra (4.5) and (4.4) in the Maxwell equation

$$
k^{2} c^{2} E_{x}=4 \pi i \omega \sigma_{x z} E_{z}
$$

we obtain

$$
E_{x} \simeq i E_{z}
$$

Therefore, exactly as in helicon waves with $k R \ll 1$ [3], the transverse part of the electric field of the wave (4.5) is circularly polarized. The magnitude of the longitudinal field $E_{y}$ should be found from the relationship (3.13). Since the magnitude $G$ is small in the case (3.16), then

$$
E_{y}=E_{z} / 2 \phi \alpha_{n} .
$$

Using the wave spectrum (4.5), its existence conditions (3.1), (4.1) and (4.3) may be written as

$$
\frac{v}{\Omega} \ll \varphi \ll \frac{1}{\alpha_{n}} \ll 1 .
$$

* We take this opportunity to correct an error made in [3] in the determination of the wave damping. Formula (4.7) should be used instead of the damping $\Gamma=\operatorname{Re} \sigma_{z z} / 2\left|\sigma_{x z}\right|$ presented in [3]. 


$$
\omega_{n} \ll v .
$$

When the inequality $v \ll \phi \Omega$ is violated, even the minimum value of the relative damping $\Gamma_{n}$ becomes large or on the order of unity, and the wave attenuates rapidly. For the customary metals $\left(n \sim 10^{22} \mathrm{~cm}^{-3}, m \sim 10^{-2 ?} \mathrm{~g}\right)$ conditions (4.13) are satisfied in fields $H \gtrsim 10^{3} 0 \mathrm{e}$, for angles $\phi$ on the order of a degree and for a mean free path $v / \nu$ on the order of several millimeters.

\section{High-frequency Waves with Discrete and Continuous Spectrum}

In the high-frequency range

$$
\omega>v
$$

there are more different weakly-damped waves than in the $\omega \ll v$ case. Exactly as in the lowfrequency case, the most interesting effects occur for small values of the parameter $w(3.16)$, when $\sigma_{x x}$ and $\sigma_{x z}$ are oscillating functions of $k R$. At the minimums of the oscillations the element $\sigma_{x x}$ (3.17) hence has a noticeable imaginary part, due to the imaginary part of $w$. To find the frequency spectrum of a weakly damped wave with a discrete wave-vector spectrum $k_{n}=\alpha_{n} / R$ it is necessary to solve the dispersion equation

$$
\left(\frac{k_{n}{ }^{2} c^{2}}{4 \pi \omega_{n}}\right)^{2}-\sigma_{x z}{ }^{2}\left(k_{n}\right)-i \frac{k_{n}{ }^{2} c^{2}}{4 \pi \omega_{n}} \sigma_{x x}\left(k_{n}, \omega_{n}\right)=0
$$

for $\omega_{n}$. Using (3.17), (3.18) and neglecting the small real part of $w$, we obtain

$$
\omega_{n}=\frac{2}{3} \sqrt{2 \pi}\left(\frac{v_{A}}{v}\right)^{2} \alpha_{n} 9 / 2 \frac{\varphi \Omega}{\rho_{n}}
$$

where

$$
\rho_{n}^{2}=1+\frac{4}{3} \sqrt{2 \pi}\left(\frac{v_{A}}{v}\right)^{2} \alpha_{n} 9 / 2 .
$$

In order to find the wave damping, it is necessary to retain the real part of $\sigma_{x x}$ in (5.2). Assuming the damping to be small we find

$$
\Gamma_{n}(k)=-\frac{\operatorname{Im} \omega}{\omega_{n}}=\frac{1 / 2 \operatorname{Re} \sigma_{x x}}{\left[-\operatorname{Im} \sigma_{x x}\left(k_{n}\right)+4 \pi \omega_{n} \sigma_{x z}\left(k_{n}\right) / k_{n}{ }^{2} c^{2}\right]}
$$

or

$$
\Gamma_{n}(k)=\frac{1}{\rho_{n}}\left[\frac{\nu}{\phi \Omega}+\sqrt{2 \pi \alpha_{n}} \sin ^{2}\left(k R-\alpha_{n}\right)\right] .
$$

As in the low-frequency case, the damping has a minimum for $k R=\alpha_{n}$ and increases rapidly as $\left|k R-\alpha_{n}\right|$ increases. 
The polarization of the transverse part of the wave's field should be found from the Maxwell equation

$$
k_{n}{ }^{2} c^{2} E_{x}=4 \pi i \omega_{n}\left[\sigma_{x x}\left(k_{n}, \omega_{n}\right) E_{x}+\sigma_{x z}\left(k_{n}\right) E_{z}\right] \text {. }
$$

If equation (5.2) is used, the relationship

$$
E_{x}=i E_{z}\left[1-\frac{4 \pi i \omega_{n}}{k_{n}{ }^{2} c^{2}} \sigma_{x x}\left(k_{n}, \omega_{n}\right)\right]^{-1 / 2}
$$

is obtained for the transveise components; from which it follows that the transverse part of the wave's field is elliptically polarized:

$$
E_{x}=i \rho_{n} E_{z}
$$

The magnitude of the longitudinal component $E_{y}$ is determined by (3.13), which yields in this case

$$
2 \phi \alpha_{n} E_{y}=\left[1+\frac{4}{3}\left(\frac{v_{A}}{v}\right)^{2} \alpha_{n}^{4}\right] E_{z}
$$

Compliance with conditions (3.1), (3.16), (5.1) and the condition $\Gamma<1$ is necessary for existence of the wave. Using the frequency spectrum (5.3), we write the strictest of these inequalities as

$$
\begin{gathered}
\frac{v}{\Omega \rho_{n}} \ll \varphi \ll \frac{1}{\alpha_{n}} \ll 1, \\
\left(\frac{v_{A}}{v}\right)^{2} \alpha_{n} 4 \ll \rho_{n} .
\end{gathered}
$$

Let us consider the change in the properties of the wave (5.3) with the change in the magnetic field.

(a) In the weak field domain where

$$
\left(\frac{v_{A}}{v}\right)^{2} \alpha_{n} 9 / 2<<1
$$

we have $\rho_{n} \rightarrow 1$. Hence, formulas $(5.3),(5.6),(5.9)$ and $(5.10)$, defining the wave spectrum, damping, and polarization, agree with formulas (4.5), (4.7), (4.11) and (4.12). In other words, in this case the high-frequency field is completely analogous, in its properties, to the low-frequency wave. The only difference is that the additional constraint (5.12) on the magnetic field and the quantity $\alpha_{n}$ appears. Hence, the existence of a wave with the discrete spectrum (4.5) is independent of the relationship between $v$ and $\omega$.

(b) In the domain of stronger fields, when the inequality (5.12) is replaced by the reverse, and

$$
\rho_{n} \gg 1 \text {. }
$$


we have

$$
\begin{gathered}
\omega_{n}=\left(\frac{2 \pi}{9}\right)^{1 / 4} \frac{v_{A}}{v} \alpha_{n} 9 / 4 \phi \Omega, \\
\Gamma(k)=\frac{1}{2 \omega_{n}}\left[v+\phi \Omega \sqrt{2 \pi \alpha_{n}} \sin ^{2}\left(k R-\alpha_{n}\right)\right], \\
E_{y}=\frac{\Omega}{3 i \omega_{n}}\left(\frac{v_{A}}{v}\right)^{2} \alpha_{n}^{3} E_{x}, \quad E_{z}<E_{x} .
\end{gathered}
$$

The transverse part of the field in this wave is polarized along the $x$ axis. Its spectrum and damping are determined only by the quantity $\sigma_{x x}$. The existence of this high-frequency wave is due to the fact that for $k R=\alpha_{n}$ the dissipative conductivity decreases radically and becomes less than the non-dissipative:

$$
\operatorname{Re} \sigma_{x x}^{\min } \ll-\operatorname{Im} \sigma_{x x}
$$

(c) In the limiting high-frequency case (5.1); the real part of the element $\sigma_{z z}($ see (3.10)) is small compared with its imaginary part, which is negative. Therefore, the dielectric constant in the $z$ direction

$$
\varepsilon_{z z}=\frac{4 \pi i \sigma_{z z}}{\omega}=6 \pi \frac{n_{0} e^{2}}{m\left(k_{z} v\right)^{2} k R}\left(1+i \frac{v}{\omega}\right)
$$

is mainly real and positive. This circumstance should lead to the existence of a weakly-damped high-frequency wave. However, because of the notable non-diagonal conductivity $\sigma_{x z}$, a sufficiently strong coupling generally exists between the two transverse waves. Since the element $\sigma_{x z}$ is a sharply oscillating function of $k R$ in the $|w|<<1$ case, this coupling is quite attenuated for

$$
\begin{gathered}
k R=\beta_{N} \equiv\left(N+\frac{3}{4}\right) \frac{\pi}{2} \\
N=0,1,2, \ldots
\end{gathered}
$$

If $k R=\beta_{N}$ then

$$
\frac{\left(\sigma_{x z}^{\min }\right)^{2}}{\sigma_{x x}\left|\sigma_{z z}\right|} \sim \frac{|v-i \omega|}{k_{z} v} \ll 1
$$

and the term $\sigma_{x z}^{2}$ may be neglected in (2.9).

Hence, the coupling between both the waves is destroyed for $k=\beta_{n} / R$, and the dispersion 
equation (2.9) decomposes into two equations. One of them yields a damped wave (the element $\sigma_{x x}$ is real for $k=k_{N}$ ). The other

$$
k_{N}^{2} c^{2}=4 \pi i \omega \sigma_{z z}\left(k_{N}, \omega\right)
$$

yields a weakly-damped wave with the discrete spectrum

$$
\omega_{N}=\left(\frac{2}{3}\right)^{1 / 2} \frac{v_{A}}{v} \beta_{N} 5 / 2 \varphi \Omega, \quad k_{N}=\frac{\beta_{N}}{R} .
$$

The relative damping of this wave is

$$
\Gamma(k)=-\frac{\operatorname{Im} \omega}{\omega_{N}}=\frac{\operatorname{Re} \sigma_{z z}+\sigma_{x z}{ }^{2} / \sigma_{x x}}{2\left|\operatorname{Im} \sigma_{z z}\right|}=\frac{1}{2 \omega_{N}}\left[\nu+\frac{\phi \Omega}{\pi} \frac{\sin ^{2} 2\left(k R-\beta_{N}\right)}{1-\sin 2 \beta_{N}}\right] .
$$

The width of the domains in $k$-space in which the wave (5.22) is weakly-damped $(\Gamma \ll 1)$ is, bounded by the condition

$$
\left(k R-\beta_{N}\right)^{2}<\frac{\omega_{N}}{\varphi \Omega}
$$

The polarization of the electric field in the wave is linear:

$$
E_{z}=2 \phi \beta_{N} E_{y}, \ldots \quad E_{x} \ll E_{z} .
$$

The domain of existence of this wave with the discrete spectrum is determined by inequalities $(3.1),(5.1)$ and the condition

$$
\frac{\left(\sigma_{x z}^{\max }\right)^{2}}{\sigma_{x x}\left|\sigma_{z z}\right|} \gg 1
$$

The strictest of these conditions may be represented as

$$
\begin{gathered}
\frac{v}{\varphi \Omega} \ll \frac{v_{A}}{v} \beta_{N} 5 / 2 \ll 1, \\
\Phi \ll \frac{1}{\beta_{N}} \ll 1 .
\end{gathered}
$$

(d) Finally, if the inequality

$$
\Phi<\frac{\omega}{\Omega}
$$

is satisfied in addition to (3.1) and (5.1), the degree of wave coupling turns out to be weak (the inequality (5.26) is replaced by the reverse for all values of $k R$, excepting small neighborhoods of the points $k R=\alpha_{n}$ at which diminution of $\sigma_{x x}$ occurs). Equation (5.21) now becomes valid for any $k R$, and the wave spectrum is converted from discrete into continuous:

$$
\omega(k)=\left(\frac{2}{3}\right)^{1 / 2} \frac{v_{A}}{v}(k R)^{5 / 2} \phi \Omega .
$$


The conditions $(5.1),(5.28)$ and the inequalities

$$
1 \ll\left(\frac{v}{v_{A}}\right)^{2 / 5} \cdot\left(\frac{\Phi \Omega}{\omega}\right)^{3 / 5}<\frac{\Omega}{\omega} \text {, }
$$

obtained from (3.1) if $k$ is expressed in terms of $\omega$ by using (5.29), should be satisfied for the wave (5.29), to exist.

The analysis made of the possibilities of the propagation of weakly-damped electromagnetic waves is based on utilizing (3.10) for the elements of the conductivity tensor. These asymptotic formulas have been obtained for a simply-connected spherical Fermi surface. It is evident enough that the nature of the asymptotic representation of the tensor $\sigma_{i j}$, particularly the derivation of the existence of sharp oscillations in the elements $\sigma_{x x}$ and $\sigma_{x z}$, remains valid even in the case of a non-spherical but simply-connected and convex Fermi surface. The difference is that $k D$, where $D$ is the diameter of the central section of the Fermi surface along the [k, $\mathbf{H}]$ direction, will replace the $2 k R$ in the arguments of the oscillating functions in (3.17), (3.18). Moreover, the dissipative current associated with the Landau damping will be directed along the electron velocity $\partial \varepsilon(\mathbf{p}) / \partial \mathbf{p}$ at the point $\mathbf{p} \cdot \mathbf{H}=0, \quad \mathbf{k} \cdot \mathbf{v}=\mathbf{0}$ on the Fermi surface $\varepsilon(\mathbf{p})=\mu$.

There results from the considerations expounded in the introduction that the amplitude of the conductivity oscillations will be relatively small in the case of a multiply-connected or non-convex Fermi surface. Indeed, in this case there are several groups of "effective" electrons moving cophasally with the wave and satisfying the condition $k_{z} \bar{v}_{z}=\omega$, rather than just one. Hence, $\sigma_{x x}$ will be the sum of expressions of type (3.17) for the different groups

$$
\sigma_{x x} \sim \sum_{j} a_{j}\left(1-\sin k D_{j}\right) .
$$

This quantity will not vanish for any real values of the wave vector $\mathbf{k}$. Hence, in the case of a multiply-connected, or non-convex Fermi surface, there is no wave with a discrete spectrum. Only a weakly damped wave with a continuous spectrum (5.29) may exist in a metal if all the dissipative currents from the different groups of "resonant" electrons are collinear. Hence, the predicted wéakly-damped waves with discrete spectrum may be observed only in metals with a simply-connected and convex Fermi surface. Alkali metals, as weil as some other, indium, say, comply with this requirement.

\section{Excitation of Waves with Discrete Spectrum. Resonance Effect}

In this section we shall consider the excitation of waves with discrete frequency and wavevector spectrum by an external electromagnetic field. Skin-effect occurs upon the incidence of an external wave on a metal surface. The acutely inhomogeneous distribution of the high-frequency field in the metal may be represented as the superposition of monochromatic plane waves with different values of $k$. Because of the shallow depth of the skin-layer, always to be found among such monochromatic waves are those whose wave vector satisfies the condition $k R=\alpha_{n}$. Resonant excitation of the proper oscillations will occur each time the frequency of the external field $\omega$ agrees with one of the frequencies $\omega_{n}$ of the proper oscillations. For a fixed frequency $\omega$ resonant excitation of waves with a discrete spectrum will occur for those values of the magnetic field $H=H_{n}$ for which $\omega_{n}(H)=\omega$.

As an example, let us consider the excitation of waves with the spectrum (5.14), in which 
the transverse part of the electric field is polarized along the $x$ axis. The resonant value of the permanent magnetic field may be written for these waves as

$$
H_{n}=\left(\frac{9}{2 \pi}\right)^{1 / 8} \alpha_{n}-9 / 8 \frac{m c}{e}\left(\frac{\omega \omega_{0} v}{\phi c}\right)^{1 / 2}
$$

It follows from (6.1) that the resonant effect is periodic in the function $H^{-8 / 9}$. Upon exciting waves with the spectrum (4.5) $H_{n} \sim \alpha_{n}{ }^{-3 / 2}$. Hence, in contrast to all the other oscillating and resonance phenomena, this resonance is neither periodic in the direct nor the reverse magnetic field.

According to [3], the distribution of the high-frequency field $\mathscr{E}_{x}(\zeta)$ and the surface impedance $Z_{x x}$ are determined by the formulas:

$$
\begin{gathered}
\mathscr{E}_{x}(\zeta)=T_{x x}(\zeta) \mathscr{E}_{x}^{\prime}(0), \\
Z_{x x}=\frac{4 \pi i \omega}{c^{2}} T_{x x}(0), \\
T_{x x}=-\frac{2}{\pi} \int_{0}^{\infty} \frac{d k \cos k \zeta}{k^{2}-4 \pi i \omega \sigma_{x x}(k) c^{-2}},
\end{gathered}
$$

where the prime denotes the derivative with respect to $\zeta$. We neglect the influence of electron scattering by the boundary of the metal (see [3]), which is not henceforth essential.

Let us first find the field $\mathscr{E}_{x}(\zeta)$ at long ranges from the metal surface $\zeta=0$. This field is characterized by the function

$$
T_{x x}(\zeta)=-\frac{2}{\pi} \int_{0}^{\infty} \frac{d k k^{5 / 2} \cos k \zeta}{k^{9 / 2}-\left(H_{n} / H\right)^{4} k_{n} 9 / 2[1+2 i \Gamma(k)]}
$$

where $k_{n}=\alpha_{n} / R$, and the expression for $\Gamma(k)$ is determined by (5.15) in which $\omega_{n}$ must be replaced by $\omega$. Let us introduce the "detuning" of resonance

$$
\Delta=\frac{8}{9} \frac{H-H_{n}}{H_{n}} \quad(|\Delta| \ll 1)
$$

and the new integration variable

$$
T=\left(\frac{k}{k_{n}}\right)^{1 / 2} \text {. }
$$

In the resonance domain $(\Delta-0)$ a small neighborhood of the pole of the integrand near the point $\tau=1$ on the complex $T$ plane, introduces the fundamental contribution. Hence, the function $T_{x x}(\zeta)$ may be represented as

$$
T_{x x}(\zeta)=-\frac{2}{\pi k_{n}} \int_{0}^{\infty} d \tau \frac{\tau^{6}\left[\exp \left(i k_{n} \zeta \tau^{2}\right)+\exp \left(-i k_{n} \zeta \tau^{2}\right)\right]}{\tau^{9}-\left[1-\frac{9}{2} \Delta+\frac{9}{4} i \Gamma(\Delta)\right]}
$$


where

$$
\Gamma(\Delta)=\frac{1}{2 \omega}\left[\nu+(2 \pi)^{1 / 2} \phi \Omega \Delta^{2}\right] .
$$

Let us rotate the contour of integration in the first member in (6.8) to the line $\arg T=\pi / 4$, and to the line arg $\tau=-\pi / 4$ in the second member. We hence obtain the sum of residues and integrals along the lines $\arg \tau= \pm \pi / 4$. For large $\zeta$ the residue of the first integral at the point

$$
\tau_{0}=1-\frac{\Delta}{2}+\frac{i}{4} \Gamma(\Delta) \text {. }
$$

yields the fundamental contribution to $T_{x x}(\zeta)$.

Hence, in the neighborhood of resonance $\Gamma(\Delta) \ll 1$ we have

$$
T_{x x}(\zeta) \simeq-\frac{4 i}{9} \frac{R}{\alpha_{n}\left[1-\Delta+\frac{i}{2} \Gamma(\Delta)\right]} \exp \left\{i \alpha_{n} \frac{\zeta}{R}\left[1-\Delta+\frac{i}{2} \Gamma(\Delta)\right]\right\}
$$

and the field at long ranges from the metal surface represents an electromagnetic wave with the length

$$
\lambda_{n}=\frac{2 \pi R}{\alpha_{n}(1-\Delta)} \approx \frac{2 \pi R_{n}}{\alpha_{n}}\left(1-\frac{\Delta}{8}\right) .
$$

Furthermore, from (6.3) and (6.5) for $\zeta=0$ and $\Delta \rightarrow 0$ we find the resonant values of the surface impédance

$$
Z_{x x}^{(n)}=\frac{16 \pi}{9} \frac{\omega R_{n}}{\alpha_{n} c^{2}}\left(1-i \operatorname{ctg} \frac{2 \pi}{9}\right), \quad R_{n}=\frac{m c v}{e H_{n}} .
$$

The magnitude of $Z_{x x}^{(n)}$ grows slightly as $n$ increases:

$$
Z_{x x}^{(n)} \sim \alpha_{n}^{1 / 8}
$$

This growth in the resonant values of the impedance as the magnetic field diminishes is due to the increase in the wavelength (6.12) of the proper electromagnetic oscillations. The width of the resonant maximum is determined by the wave damping and agrees, in order of magnitude, with the relative width of the domain in $k$-space in which the wave damps weakly

$$
\Delta_{n}=\frac{\Delta H}{H_{n}} \sim\left(\frac{\omega}{\phi \Omega_{n} \sqrt{\alpha_{n}}}\right)^{1 / 2}
$$

The absolute width $\Delta H$ decreases with the number as $n^{-13 / 16}$.

For comparison, let us consider the surface impedance of the metal far from resonance, when $\Gamma(\Delta) \gg 1$. In this case the expression for $\mathbf{T}_{x x}(0)$ may be written as

$$
T_{x x}(0)=-\frac{2}{\pi} \int_{0}^{\infty} \frac{k^{2} d k}{k^{4}-i \delta_{\mathrm{eff}}^{-4}(1-\sin 2 k R)} .
$$


where

$$
\delta_{\mathrm{eff}}=\left(\frac{2 v^{2} c^{2} \varphi}{3 \omega_{0}^{2} \omega \Omega}\right)^{\mathrm{x}}
$$

is the effective depth of penetration of the external electromagnetic field in the metal. The quantity $T_{x x}(0)$ has been evaluated by one of the authors [6]. According to $[6]$, for $\delta_{e} f<<R$

$$
Z_{x x}^{\text {nones }}=\left(\frac{2}{\pi}\right) \gamma\left(\frac{1}{4}\right) \frac{\omega \delta_{\text {eff }}}{c^{2}} \exp \left(\frac{3 \pi i}{8}\right) \sim \omega^{*} H_{-K} / 4
$$

where $\gamma(x)$ is the Euler gamma function.

Hence, narrow resonance maximums, corresponding to resonant excitation of proper oscillations with spectrum (5.14), are superposed on the smooth dependence of the impedance on the magnetic field (6.18). The ratio of the resonant value of the impedance $Z_{x x}^{(n)}$ to the nonresonant value $(6.18)$ is

$$
\left|\frac{Z_{x x}^{(n \cdot)}}{Z_{x x}}\right| \quad \approx \frac{R_{n}}{\alpha_{n} \delta_{\text {eff }}} \approx\left(\frac{\phi \Omega_{n}}{\omega} \sqrt{\alpha_{n}}\right)^{1 / 4}>1 \text {, }
$$

i.e. far from resonance the surface impedance turns out to be several times smaller. The dependence of the impedance on the reciprocal magnetic field is shown schematically in Fig. 2.

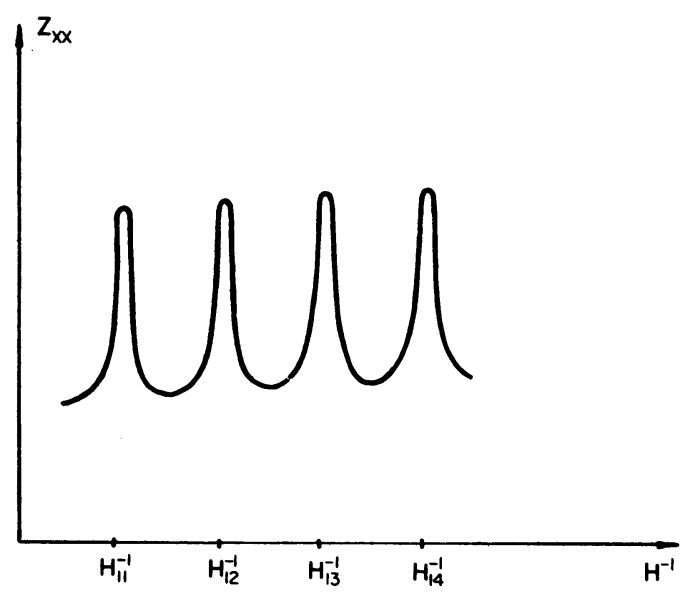

FIGURE 2

The dependence of the impedance of a metal on $H$ is analogous even for the excitation of other weakly-damped electromagnetic waves with discrete spectrum.

\section{Appendix}

Let us find the asymptotic form of the elements $\sigma_{x \beta}(\beta=x, \eta)$ in the limiting case (3.1). Utilizing (3.9), the expression (3.8) for these elements may be represented as: 


$$
\begin{gathered}
\sigma_{x x}=\frac{\sigma}{\pi} \int_{0}^{\pi} d \theta \frac{\sin ^{2} \theta}{u+i \cos \theta}[1-\sin (2 \alpha \sin \theta)], \\
\sigma_{x \eta}=\frac{\sigma}{\pi} \int_{0}^{\pi} d \theta \frac{i \cos \theta \sin \theta}{u+i \cos \theta} \cos (2 \alpha \sin \theta),
\end{gathered}
$$

where

$$
\begin{gathered}
\sigma=\frac{3 n_{0} e^{2}}{2 m k_{z} v k R}, \\
\alpha=k R, \quad u=(v-i \omega) / k_{z} v,
\end{gathered}
$$

and

$$
|u| \ll 1 \ll \alpha
$$

Let us introduce the new integration variable $\mu=\cos \theta$ and let us write (A.1) and (A.2) as

$$
\begin{gathered}
\sigma_{x x}=\frac{\sigma}{\pi} \int_{-1}^{1} d \mu \frac{\left(1-\mu^{2}\right)^{1 / 2}}{u+i \mu}-\sigma F, \\
\sigma_{x \eta}=\frac{\sigma}{\pi} \int_{-1}^{1} d \mu \cos \left[2 \alpha\left(1-\mu^{2}\right)^{1 / 2}\right]-\sigma u G,
\end{gathered}
$$

where

$$
\begin{gathered}
F=\frac{1}{\pi} \int_{-1}^{1} d \mu \frac{\left(1-\mu^{2}\right)^{1 / 2}}{u+i \mu} \sin \left[2 \alpha\left(1-\mu^{2}\right)^{1 / 2}\right], \\
G=\frac{1}{\pi} \int_{-1}^{1} d \mu \frac{\cos \left[2 \alpha\left(1-\mu^{2}\right)^{1 / 2}\right]}{u+i \mu}
\end{gathered}
$$

The first member in (A.6) is $\sigma$, to the accuracy of small terms on the order of $u$. The expression for the first member in (A.7) may be found by using the method of stationary phase. Its principal term for $\alpha \gg 1$ is

$$
(\pi \alpha)^{-1 / 2} \cos (2 \alpha-\pi / 4)
$$

Let us now find the asymptotic formulas for the functions $F$ and $G$. Because of the inequalities (A.5) the domain of small values of $\mu$ introduces the fundamental contribution to $F$ and $G$. For 
this reason all the smooth functions of $\mu$ may be expanded in power series in $\mu$, and the limits of integration may be considered infinite. After the change of variable

$$
\mu=\tau \alpha^{-1 / 2}
$$

the formulas for $F$ and $G$ may be written as:

$$
\begin{aligned}
& V \equiv G+i F=\frac{1}{\pi} \int_{-\infty}^{\infty} \frac{d \tau}{w+i \tau} \exp i\left(2 \alpha-\tau^{2}\right), \\
& w=u \alpha^{1 / 2}, \quad F=\operatorname{Im} V, \quad G=\operatorname{Re} V,
\end{aligned}
$$

where the parameter $w$ must be considered real for the real and imaginary parts taken in (A.12). The pole of the integrand in (A.11) is found at the point $\tau_{0}=i w$, where $\operatorname{Re} \tau_{0}$ and $\operatorname{Im} \tau_{0}$ are positive. Hence, the contour of integration in (A.11) may be combined with the line arg $T=-\pi / 4$. After the substitution $\tau=t \exp (-\pi i / 4)$, we reduce (A.11) to

$$
\begin{aligned}
V & =\frac{1}{\pi i} \int_{-\infty}^{\infty} \frac{d t}{t+w \exp (-\pi i / 4)} \exp \left(2 i \alpha-t^{2}\right) \\
& =\frac{1}{\pi i} \int_{-\infty+w e^{-\pi i / 4}}^{\infty+w e^{-\pi i / 4}} \frac{d z}{z} \exp \left[2 i \alpha-\left(z-w e^{-\pi i / 4}\right)^{2}\right] .
\end{aligned}
$$

in which $\operatorname{Im} w \exp (-\pi i / 4)<0$.

Let us displace the contour of integration to the real axis in the complex $z$ plane. The integral over a small semi-neighborhood around the point $z=0$ equals the residue of the integrand, and the integral over the real axis must be taken in the sense of the principal value:

$$
V=\exp i\left(2 \alpha+w^{2}\right) \quad\left\{1+\frac{1}{\pi i} f_{-\infty}^{\infty} \frac{d x}{x} \exp \left(-x^{2}+2 x w e^{-\pi i / 4}\right)\right\}
$$

The integral in the braces in (A.14) is easily reduced to the probability integral by differentiation with respect to the parameter $w$ and subsequent integration. Finally, we obtain

$$
V=\exp (2 i \alpha-\pi i / 4) \frac{2}{\pi^{1 / 2}} \int_{0}^{\infty} d x \exp \left(i x^{2}-2 w x\right)
$$

It is seen in (A.15) that in the limiting case

$$
|w|<1 \quad V=\exp (2 i \alpha)\left[1-\frac{2 w}{\pi^{3 / 2}} \exp \left(\frac{\pi i}{4}\right)+O\left(w^{2}\right)\right] .
$$

In the opposite case

$$
|w| \gg 1 \quad V=\frac{\exp (2 i \alpha-\pi i / 4)}{\pi^{1 / 2} w}\left[1+\frac{i}{2 w^{2}}+O\left(w^{-4}\right)\right] .
$$




\section{References}

1. 0.v. Konstantinov and V. I. PEREL', J. Exp. Th. Phys. 38, 161 (1960).

2. S. BUCHSBAUM and J. GALT, Phys. Fluids 4, 1514 (1961).

3. E. A. KANER and V.G. SKOBOV, J. Exp. Th. Phys. 45, 610 (1963).

4. A.B. PIPPARD, Phil. Mag. 2, 1147 (1957).

5. E. A. Kaner, V.G. PESChANSKII and I. A. PRIVOROTSKII, J. Exp. Th. Phys. 40, 214 (1961).

6. E.A. KANER, J. Exp. Th. Phys. 44, 1036 (1963). 